\title{
Lassa Fever Case Report: Challenges in Making Early Diagnosis
}

\section{Peter Ekpunobi Chime ${ }^{1 *}$, Ethel Nkechi Chime ${ }^{2,3}$, Edmund Ndibuagu ${ }^{4}$, Fintan Chinweike Ekochin ${ }^{1}$, Sussan Arinze-Onyia ${ }^{4}$, Bibiana Oti $^{1}$}

\begin{abstract}
${ }^{1}$ Department of Medicine, Enugu State University of Science and Technology Teaching Hospital, Enugu, Nigeria ${ }^{2}$ Department of Otorhinolaryngology, University of Nigeria Teaching Hospital, Enugu, Nigeria ${ }^{3}$ Department of Otorhinolaryngology, Enugu State University of Science and Technology Teaching Hospital, Enugu, Nigeria ${ }^{4}$ Department of Community Medicine, Enugu State University of Science and Technology Teaching Hospital, Enugu, Nigeria Email: *pe4chyme@yahoo.com
\end{abstract}

How to cite this paper: Chime, P.E., Chime, E.N., Ndibuagu, E., Ekochin, F.C., Arinze-Onyia, S. and Oti, B. (2020) Lassa Fever Case Report: Challenges in Making Early Diagnosis. Advances in Infectious Diseases, 10, 228-234.

https://doi.org/10.4236/aid.2020.105020

Received: September 17, 2020

Accepted: November 1, 2020

Published: November 4, 2020

Copyright (c) 2020 by author(s) and Scientific Research Publishing Inc. This work is licensed under the Creative Commons Attribution International License (CC BY 4.0).

http://creativecommons.org/licenses/by/4.0/

\section{Open Access}

\begin{abstract}
Introduction: Lassa fever is a disease of public health importance because of the associated morbidity and high case fatality rate among hospitalized patients. Even after recovery, there may be residual problems such as sensorineural hearing loss. The initial presentation of Lassa fever may be with non-specific symptoms similar to what is seen in the more common febrile illnesses such as malaria or typhoid fever. In such a setting therefore, timely diagnosis of Lassa fever may be difficult. Case Report: We report a case of Lassa fever that presented to our institution. She was a middle aged woman who had non-specific symptoms of febrile illness and who died in less than 48 hours of admission. She had a subtle bleeding on the lip just before death which was what raised the suspicion for Lassa fever. Laboratory confirmation of Lassa fever was made retrospectively. Conclusion: When the presenting symptoms are non-specific, a high index of suspicion is required for timely recognition of Lassa fever. Early diagnosis is important for prompt therapeutic intervention as well as for limiting the spread of the disease. This is the second case of Lassa fever presenting to our hospital, but the first published case of Lassa fever from our hospital.
\end{abstract}

\section{Keywords}

Lassa Fever, Lassa Virus, Lassa Fever Diagnosis, Lassa Fever Symptoms and Signs

\section{Introduction}

Lassa fever is a disease of public health importance because of the associated 
morbidity as well as high case fatality rate in hospitalized patients. Lassa fever is one of the viral haemorrhagic fevers and it is caused by Lassa virus which is an arenavirus transmitted by Mastomysnatalensis either directly by eating rat or by taking food contaminated by rat's urine or faeces [1] [2]. Lassa fever is endemic in Nigeria and in some other West African countries such as Guinea, Benin, Mali, Ghana and Sierra Leone and Senegal [1] [2]. Lassa fever is also found outside West Africa in countries such as Central African Republic and Democratic Republic of the Congo [1]. Also, there have been reports of imported Lassa fever to other continents of the world [3] [4] [5].

Laboratory tests for Lassa fever include enzyme linked immunosorbent assay (ELISA), indirect fluorescent-antibody test (IFA), virus isolation and reverse transcriptase polymerase chain reaction (RT-PCR) [6] [7].

A number of complications can occur in Lassa fever, of which one of the disabling complications is sensorineural hearing loss. In a study done in Sierra Leone [1], a high incidence of sensorineural hearing loss was found among those suffering from Lassa fever. Sensorineural hearing loss was also found among those with serological evidence of past exposure to Lassa virus [1], indicating that hearing loss may persist in some people even after recovery from Lassa fever. Although, majority of subjects (about 80\%) with Lassa fever may have mild or no symptoms [8], in hospitalized patients, the case fatality rate may be as high as $28 \%$ [9].

The importance of timely diagnosis of Lassa fever is that both the morbidity and mortality associated with Lassa fever can be reduced and also secondary infection from the patient can be prevented. Starting ribavirin early (within the first six days) in addition to supportive care improves clinical outcome in Lassa fever [1].

Up till December 31st, 2019, no confirmed Lassa fever case had been reported from the Enugu State University of Science and Technology Teaching Hospital, Parklane, Enugu, Nigeria. Between January 2020 and February 2020, the first two cases of Lassa fever presented within a space of five weeks of each other to this hospital. The two patients died. However, no health worker was infected even though the diagnosis of Lassa fever was not made early enough. The second of the two cases is presented in this case report in order to highlight the challenges in making timely diagnosis of Lassa fever.

\section{Case Report}

On the 18th of February, 2020, a 56 year old female Nigerian who was residing in a suburb near the state capital of Enugu State was referred to our institution when her health condition did not improve following initial treatment. She gave a history of fever and generalized body weakness of a week duration, and difficulty in breathing and abdominal pains of three days duration. The fever was of high grade, intermittent, worse at night and associated with chills and rigors. There was no history of vomiting, diarrhea or bleeding from any part of the 
body. There was no history of cough, neck pain, pain on passing urine or sore throat. She was not a known hypertensive or diabetic. She first went to a private hospital where she was admitted and treated without improvement before the referral.

At presentation, her radial pulse was 100 beats per minute, respiratory rate was 28 cycles per minute and blood pressure (BP) was $80 / 50 \mathrm{mmHg}$ in the supine position. Temperature was sub normal. An initial working diagnosis of malaria to rule out enteric fever was made by the Accident and Emergency Medical Officer (Casualty Officer) who commenced her on intravenous fluids (normal saline initially and subsequently alternated normal saline with 5\% Dextrose water), treatment for malaria and intravenous ceftriaxone $1 \mathrm{gm}$ twice daily as empirical treatment for typhoid fever, pending laboratory investigations.

She was subsequently reviewed by the medical team on call. Physical examination revealed a middle aged woman who was tachypnoeic, afebrile, and had no cyanosis, no digital clubbing, no jaundice and no pedal edema. The respiratory rate was now 30 cycles per minute and her chest was clinically clear. By this time her BP had improved to $100 / 60 \mathrm{mmHg}$. The apex beat was at the 5th left intercostal space in the midclavicular line. The abdomen showed generalized tenderness with guarding. Liver, spleen and kidneys were not palpable. A working diagnosis of typhoid fever was made. Intravenous metronidazole and analgesic were added to her drug regimen. The following laboratory investigations were to be done. Full blood count, erythrocyte sedimentation rate (ESR), blood smear for malaria parasites, fasting blood glucose, liver function tests, widal test, serum electrolytes, urea and creatinine, urinalysis and urine microscopy, culture and sensitivity, stool microscopy, culture and sensitivity, blood cultures, chest X-ray and abdominal ultrasound examination.

Less than 48 hours after the patient presented at the hospital, blood was noticed around her lips. Her condition deteriorated rapidly and she died despite resuscitative measures. She died from the complications of Lassa fever. The patient's blood samples which were earlier collected for basic laboratory investigations were retrieved from the laboratory and sent for Lassa virus test and the resultant reverse transcriptase polymerase chain reaction was positive.

The infectious disease prevention and control team was alerted. Following the usual standard protocol for handling the body of a patient that died from a highly infectious disease, the team removed the dead body from the ward, de-contaminated the ward and commenced contact tracing. There was no evidence of secondary transmission from the case-patient to any healthcare worker or to other contacts traced.

\section{Discussion}

We reported a case of a middle aged woman who presented with non-specific symptoms of febrile illness in a center where malaria and typhoid fever are more common and Lassa fever is rare. She died in less than 48 hours of admission. 
This patient was seen before the arrival of COVID-19 in Nigeria. The first case of COVID-19 in Nigeria flew into Nigeria from Italy on February 25th, 2020 [10], while the first reported two cases of COVID-19 in Enugu State was on 27th March, 2020. The patient in this case report had never travelled out of Nigeria. She had had no contact with anybody from outside the country, at least, in recent times. She was a farmer living in a suburb. Therefore, the possibility of COVID-19 as a differential diagnosis was very unlikely. Multidrug resistance by malaria parasites is a problem in this part of the world. Plasmodium falciparum is the commonest malaria parasite in African countries, including Nigeria [11] and it is one of the two species of malaria parasites notorious for multidrug resistance [12]. Therefore, even if non-specific symptoms persist in a patient after malarial therapy in this part of the world, it still may not rule out the possibility of the person having malaria.

Although, the patient had fever in the early stage of her illness, fever was not prominent when she presented to our hospital and she was not febrile on examination. Bleeding which is considered to be the hallmark of Lassa fever and of other viral haemorrhagic fevers was subtle and a terminal event in this patient. A little bleeding was noticed around her lip just before death which was what raised the suspicion for Lassa fever. In one case-control study in Sierra Leone, mucosal bleeding occurred only in $17 \%$ of cases of Lassa fever patients [13]. Until the time bleeding was noticed in our patient, she was treated like any other patient rather than like a high risk patient. Fortunately, there was no secondary transmission to any hospital staff from this patient. This case illustrated some of the challenges faced by clinicians in this part of the country in making timely diagnosis of Lassa fever. It also showed the potential health risks to health staff and to others when such diagnosis is not made early enough. This was the second case of Lassa fever seen in this hospital. Enugu State has a very low incidence of Lassa fever. Between 1st of January, 2020 and 6th of September 2020, there have been only 10 confirmed cases of Lassa fever in Enugu State of Nigeria compared to 339 confirmed cases in Edo State and 363 confirmed cases in Ondo State [14].

Challenges in making timely diagnosis of Lassa fever arise from 1) late presentation of patients to centers which have the capability of managing Lassa fever cases, 2) patients presenting with nonspecific symptoms and 3) length of time required to get laboratory diagnosis. When symptoms are nonspecific, both the patient and the doctor are more likely to think of the more common febrile illness such as malaria or typhoid fever. Malaria is much more common than Lassa fever in West Africa. In Nigeria, there are only five laboratories for Lassa fever test which are distributed throughout the country. Other workers have highlighted the difficulty in making timely diagnosis of Lassa fever [1] [15]. Woyessa et al. [16] reported two cases of Lassa fever in Liberia which were misdiagnosed by the primary health physician as malaria and respiratory tract disease. The two patients died before laboratory tests confirmed them as cases of 
Lassa fever. In 2016, there was a missed case of Lassa fever who presented with fever and abdominal tenderness and who was subjected to diagnostic laparotomy in Togo [17]. The patient later died in Germany where he was transferred for further management. In 2009, a young man who visited Mali from the United Kingdom took ill and developed fever. He was thought to be suffering from malaria because of the symptoms he had and because Lassa fever had not been reported from Mali previously. However, laboratory tests confirmed that he had Lassa fever. Unfortunately, he died from the complications of Lassa fever [18]. Timely diagnosis and prompt therapeutic intervention will help in reducing the mortality associated with Lassa fever.

In Nigeria, as in other West African countries, index of suspicion for Lassa fever is especially high only during epidemics. Sensitization of the populace on the need to go to hospital early when they are sick, enhanced expertise of the healthcare workers and readily accessible diagnostic facilities will facilitate timely diagnosis of Lassa fever. If diagnosis of Lassa fever is made early in a patient, proper therapeutic intervention can be made early enough. Also, not recognizing a case of Lassa fever may cause a delay in deploying adequate prevention protocols aimed at limiting the spread of the disease to healthcare workers, to other patients as well as to patients' relatives. And this includes proper handling of the patient's body fluids including laboratory samples.

Another important challenge in the management of this patient was financial constraint on the part of the patient which made it impossible for all the desired laboratory tests to be done. This is a common problem in developing countries. Peters et al. gave a detailed discussion on the relationship between poverty and access to health care in developing countries [19].

\section{Conclusion and Recommendation}

This is the second case of Lassa fever presenting to our hospital, but the first published case of Lassa fever from our hospital. In the part of the world where malaria and other febrile illnesses are common and Lassa fever is rare, a very high index of suspicion is required to be able to recognize a case of Lassa fever early enough. Late presentation of Lassa fever patients to centers capable of managing cases of Lassa fever still remains a major challenge. Timely diagnosis of Lassa fever is important for timely institution of definitive therapy as well as for the containment of the disease.

\section{Conflicts of Interest}

The authors declare no conflicts of interest regarding the publication of this paper.

\section{References}

[1] Richmond, J.K. and Baglole, D.J. (2003) Lassa Fever: Epidemiology, Clinical Features, and Social Consequences BMJ, 327, 1271-1275. 
https://doi.org/10.1136/bmj.327.7426.1271

[2] World Health Organization (2020) Emergencies Preparedness, Response. Lassa Fever-Nigeria. Disease Outbreak News 20 February 2020.

https://www.who.int/csr/don/20-february-2020-lassa-fever-nigeria/en

[3] Kitching, A., Addiman, S., Cathcart, S., et al. (2009) A Fatal Case of Lassa Fever in London, January 2009. Eurosurveillance, 14, 19117.

[4] Amorosa, V., MacNeil, A., McConnell, R., Patel, A., et al. (2010) Imported Lassa Fever, Pennsylvania, USA. Emerging Infectious Diseases, 16, 1598-1600. https://doi.org/10.3201/eid1610.100774

[5] Kofman, A., Choi, M.J. and Rollin, P.E. (2019) Lassa Fever in Travelers from West Africa, 1969-2016. Emerging Infectious Diseases, 25, 236-239. https://doi.org/10.3201/eid2502.180836

[6] Niklasson, B.S., Jahrling, P.B. and Peters, C.J. (1984) Detection of Lassa Virus Antigens and Lassa Virus-Specific Immunoglobulins $\mathrm{G}$ and $\mathrm{M}$ by Enzyme-Linked Immunosorbent Assay. Journal of Clinical Microbiology, 20, 239-244. https://doi.org/10.1128/JCM.20.2.239-244.1984

[7] Bausch, D.G., Rollin, P.E., Demby, A.H., et al. (2000) Diagnosis and Clinical Virology of Lassa Fever as Evaluated by Enzyme-Linked Immunosorbent Assay, Indirect Fluorescent-Antibody Test, and Virus Isolation. Journal of Clinical Microbiology, 38, 2670-2677. https://doi.org/10.1128/JCM.38.7.2670-2677.2000

[8] World Health Organization. Lassa Fever Fact Sheet. http://www.who.int/mediacentre/factsheets/fs179

[9] Inegbenebor, U., Okosun, J. and Inegbenebor, J. (2010) Prevention of Lassa Fever in Nigeria. Transactions of the Royal Society of Tropical Medicine and Hygiene, 104, 51-54. https://doi.org/10.1016/j.trstmh.2009.07.008

[10] The Guardian (2020) Nigeria Confirms First Case of Coronavirus. 28 February 2020. https://guardian.ng/news/nigeria-confirms-first-case-of-coronavirus

[11] World Health Organization (2019) The "World Malaria Report 2019" at a Glance. https://www.who.int/news-room/feature-stories/detail/world-malaria-report-2019

[12] Centre for Disease Control and Prevention (2020) Drug Resistance in the Malaria-Endemic World.

https://www.cdc.gov/malaria/malaria_worldwide/reduction/drug_resistance.html

[13] McCormick, J.B., King, I.J., Webb, P.A., Johnson, K.M., et al. (1987) A Case-Control Study of the Clinical Diagnosis and Course of Lassa Fever. The Journal of Infectious Diseases, 155, 445-455. https://doi.org/10.1093/infdis/155.3.445

[14] Nigeria Centre for Disease Control (2020) An Update of Lassa Fever Outbreak in Nigeria. S/No 1. An Update of Lassa Fever Outbreak in Nigeria for Week 33. https://ncdc.gov.ng/diseases/sitreps/?cat=5\&name=An\%20update\%20of\%20Lassa $\%$ 20fever\%20outbreak\%20in\%20Nigeria

[15] Ibekwe, T. (2012) Lassa Fever: The Challenges of Curtailing a Deadly Disease. The Pan African Medical Journal, 11, 55.

[16] Woyessa, A.B., Maximore, L., Keller, D., et al. (2019) Lesson Learned from the Investigation and Response of Lassa Fever Outbreak, Margibi County, Liberia, 2018: Case Report. BMC Infectious Diseases, 19, 610.

https://doi.org/10.1186/s12879-019-4257-Z

[17] Lehmann, C., Kochanek, M., Abdulla, D., et al. (2017) Control Measures Following a Case of Imported Lassa Fever from Togo, North Rhine Westphalia, Germany, 2016. Eurosurveillance, 22, pii=17-00088. 
https://doi.org/10.2807/1560-7917.ES.2017.22.39.17-00088

[18] Atkin, S., Anaraki, S., Gothard, P., Walsh, A., Brown, D., Gopal, R., Hand, J. and Morgan, D. (2009) The First Case of Lassa Fever Imported from Mali to the United Kingdom, February 2009. Eurosurveillance, 14, pii. 19145.

[19] Peters, D.H., Garg, A., Bloom, G., et al. (2008) Poverty and Access to Health Care in Developing Countries. Annals of the New York Academy of Sciences, 1136, 161-171. https://doi.org/10.1196/annals.1425.011 